\title{
Property Analysis and Experimental Study of the Broadband Transmis- sion-Line Transformer in Multimode Feed Network
}

\author{
Zhan Huawei ${ }^{*}$ Liu Weina, Li Qiaoyu, Yan Tingting and ZhengJie \\ College of Physics and Electronic Engineering, Henan Normal University, Xinxiang, Henan, 453007, P.R. China
}

\begin{abstract}
Transmission-line transformers are circuits useful for microwave impedance matching applications due to their broad operating bandwidth. Multimode feed network is composed of two substructures, which are constituted by the transmission-line transformer. Beginning with the broadband transmission-line transformer with 4:1 impedance transformation, supposing the currents on the two lines are not equal but opposite and with the application of two line transmission-line theory, the current-voltage relationships of the asymmetrical (current) bifilar even transmission-line are obtained. An equivalent model with mutual coupling between the subject transmission-lines has been proposed, and its characteristics for impedance transformation have been analyzed. Also, a useful and effective analytic method for bifilar transmission-line transformer has been proposed. The calculated values are in good agreement with the metrical values. So in real application it can better improve the performance of the component and can be used more efficiently.
\end{abstract}

Keywords: Transmission-line transformer, Multimode feed network, Input impedance.

\section{INTRODUCTION}

The multimode feed network of multi-mode multi-feed shortwave antenna is composed of impedance transformer and isolator [1]. The function of impedance transformer is the impedance match. The function of isolator is to divide (or synthesize) the power and isolate the signal. Both the two substructures are constituted by the transmission-line transformer, so they can be analyzed by the method of analyzing transmission-line transformer; the equivalent circuits are shown in Fig. (1). In the view of substructure cascade, the characteristic of feed network can be gained through the characteristic of impedance transforming substructure and isolating substructure.

In 1959, based on the hypothesis of equal but opposite currents on the two lines, transmission-line equation was first applied by Ruthroff to analyze the bifilar 1:4 transmission-line transformer. And the input impedance of the bifilar 1:4 transmission-line transformer was obtained but not found suitable at low frequency [2]. Abrie verified that different currents in the two line conducts must be considered [3]. Some scientists analyzed transmission-line transformer by applying electromagnetism coupling coefficients and even and odd-mode currents [4].

In this paper, supposing the currents on the two lines are not equal but opposite and referring to the transmission-line equation, a four-end network model for the asymmetrical (current) bifilar even transmission line is obtained. So a method which holds for bifilar even transmission-line transformer at both low frequency and high frequency is put forward. This paper also presents an analysis of substructure by this method [5]. The result correctly demonstrates the effect

*Address correspondence to this author at the College of Physics and Electronic Engineering, Henan Normal University, Xinxiang, Henan, 453007, P.R. China; Tel: 13937337544; E-mail: zhanhw@126.com of $\mathrm{Lp}$ (magnetizing inductance) at low frequency and fits into the result gotten with the application of transmission-line equation at high frequency.
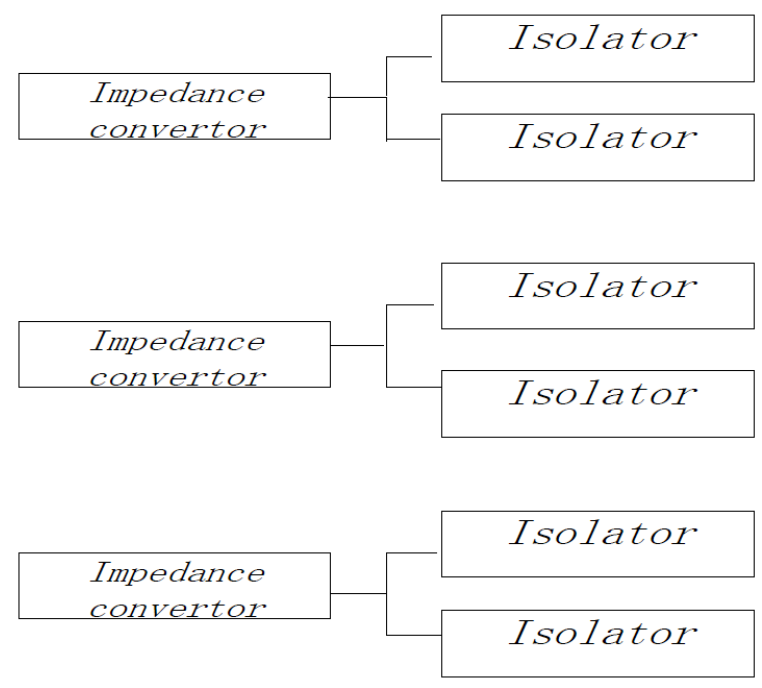

Fig. (1). The feed network configuration.

\section{ANALYSIS OF TRANSMISSION LINE TRANS- FORMER}

The basic expression for the input impedance of a transmission-line transformer Fig. (2) was first obtained by Ruthroff:

$$
Z_{\text {in }}=\frac{R_{0}\left\{2 R_{L}[1+\cos (\beta l)]+j R_{0} \sin (\beta l)\right\}}{R_{0} \cos (\beta l)+j R_{L} \sin (\beta l)}
$$




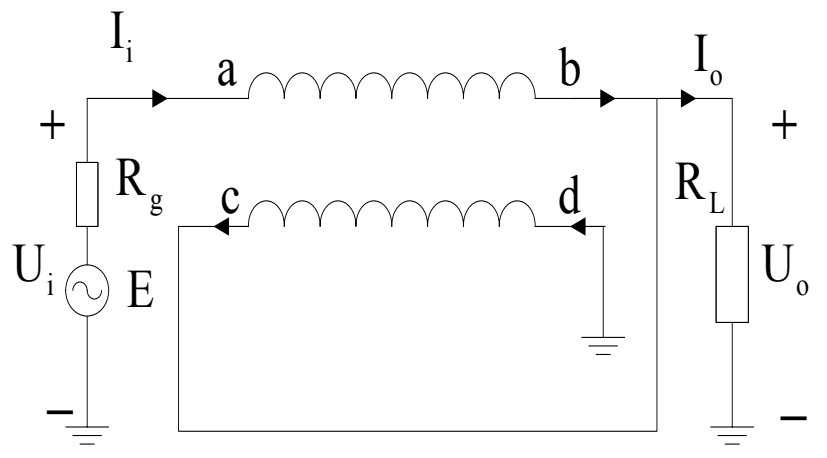

Fig. (2). The equivalent model of 4:1 TLT.

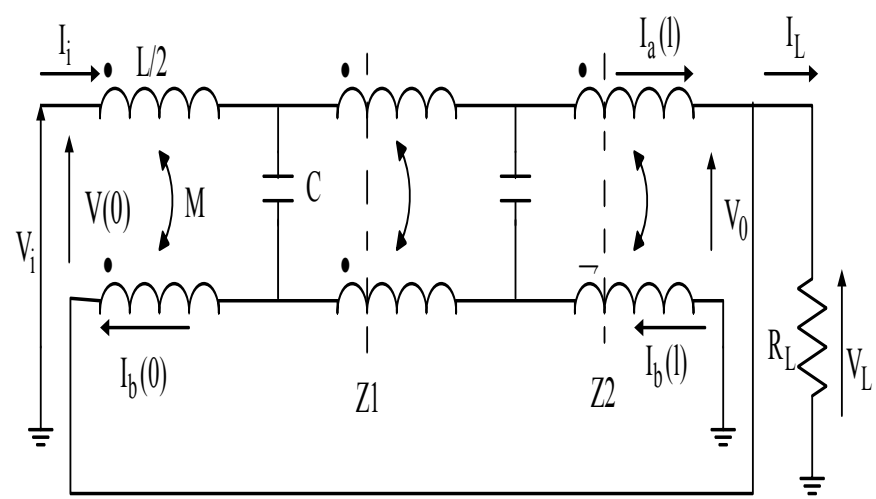

Fig. (3). The equivalent circuit model of TLT.

Where:

$\beta=\omega(L C)^{0.5}$

$\omega=$ the radian frequency,

$l=$ the electrical length of the transmission-line,

$\mathrm{R}_{0}=$ the characteristic impedance of the transmission-line,

$\mathrm{R}_{\mathrm{L}}=$ the load impedance,

$\mathrm{L}$ and $\mathrm{C}=$ the resonant inductance and capacitance, respectively.

If $\ell<<\lambda, \cos \beta l \approx 1, \sin \beta l \approx 0$, then $Z_{\text {in }}=4 R_{L}$. With this expression, an input impedance of approximately four times the load impedance is obtained at the design frequency. The formula is based on the usual hypothesis of equal but opposite currents on the two lines [6]. Recently, Abrie verified that different currents in the two line conducts must be considered; Fig. (3). shows the electrical model used for the transmission-line transformer analysis. Inductance L/2 and mutual inductance $\mathrm{M}$ are related to the system geometry. Since the currents in the two line conductors are not equal, the balanced and unbalanced components should be considered. The inductance seen by the unbalanced currents (which are also called coil-mode currents) will differ from that of the transmission-line, as the effect of $M$ is a function of the current verse (or direction).
In the case of equal-verse currents, the equivalent inductance is given by:

$\mathrm{L}_{\text {coil }}=\mathrm{L}+2 \mathrm{M}$

Note that the network appears to be a coil to the equalverse currents. In the case of equal but opposite currents, the equivalent inductance is given by:

$\mathrm{L}_{\text {line }}=\mathrm{L}-2 \mathrm{M}$

In the practical case where a transmission-line is wound on a toroid, the parameters to be considered are the transmission-line inductance (given by $\mathrm{L}_{\text {line }}$ ) and the toroid inductance (described by $\mathrm{L}_{\text {coil }}$ ):

$\mathrm{L}_{\text {line }}=0.92 \ell \mu_{r} \lg (m / r)$

$\mathrm{L}_{\text {coil }}=\mu N^{2} r_{c}^{2} / 2 R$

Where:

$\ell=$ the line length $(\mathrm{m})$,

$\mu_{\mathrm{r}}=$ the relative permeability,

$\mathrm{m}=$ the spacing between the centers of the wires $(\mathrm{m})$,

$\mathrm{r}=$ the radius of the wires $(\mathrm{m})$,

$\mathrm{r}_{\mathrm{C}}=$ the radius of the coil, 
$\mathrm{N}=$ the number of turns,

$\mathrm{R}=$ the radius of the toroid(m), and

$\mu=$ the permeability of the medium inside the coil.

Using the hypothesis $\mathrm{L}_{\text {coil }}>\mathrm{L}_{\text {line }}$ from Eqs. (2) and (3), it is determined that: $0<\mathrm{M}<\mathrm{L} / 2$ Using Eqs. (2) and (3), it is possible to define: $\mathrm{K}=\mathrm{M} /(\mathrm{L} / 2)$.

Based on Fig. (3), the following differential equations are obtained:

$\frac{d V}{d Z}=(L / 2)(K-1) \frac{d I_{a}}{d t}+(L / 2)(K-1) \frac{d I_{b}}{d t}$

$\frac{d I_{a}}{d Z}=-C \frac{d V}{d t}$

$\frac{d I_{b}}{d Z}=-C \frac{d V}{d t}$ as:

In the frequency domain, these solutions can be written

$I_{a}(Z, \omega)=\left(I_{i} / 2\right)[1+\cos (\mu Z)]+\left[I_{b}(0) / 2\right][\cos (\mu Z)-1]-$

$\left[j\left(V_{i}-V_{o}\right) /\left(L^{0.5} / C^{0.5}\right)(1-K)^{0.5}\right] \times \sin (\mu Z)$

$V(Z, \omega)=\left(V_{i}-V_{o}\right) \cos (\mu Z)-\left[j\left(L^{0.5} / C^{0.5}\right)(1-K)^{0.5} / 2\right]$

$\times\left[I_{i}+I_{b}(0)\right] \sin (\mu Z)$

$I_{b}(Z, \omega)=\left(I_{b}(0) / 2\right)[1+\cos (\mu Z)]+\left[I_{i} / 2\right][\cos (\mu Z)-1]$

$\left[j\left(V_{i}-V_{o}\right) /\left(L^{0.5} / C^{0.5}\right)(1-K)^{0.5}\right] \times \sin (\mu Z)$

Where: $\mu=\beta(1-K)^{0.5}, \beta=\omega(L C)^{0.5}$, and $0<K<1$.

Note that $\mathrm{L}=\left(\mathrm{L}_{\text {coil }}+\mathrm{L}_{\text {line }}\right) / 2$ here. from Fig. (3):

$V(0)=V_{i}-V_{0}, I_{o}=-I_{L}, I_{a}(0)=I_{i}$.

The circuit's impedance parameters can be written as:

$\mathrm{Z}_{11}=\frac{\mathrm{V}_{\mathrm{i}}}{\mathrm{I}_{\mathrm{i}}} \mid \mathrm{I}_{0 \rightarrow 0}=2[\cos (\mu \ell)+1]$

$/\{\mathrm{A} \sin (\mu \ell)-\mathrm{Q}[\cos (\mu \ell)+1]\}$

$\mathrm{Z}_{12}=\frac{\mathrm{V}_{\mathrm{i}}}{\mathrm{I}_{0}} \mid \mathrm{I}_{\mathrm{i} \rightarrow 0}=[\cos (\mu \ell)+1]$

$/\{A \sin (\mu \ell)-\mathrm{Q}[\cos (\mu \ell)+1]\}$

$\mathrm{Z}_{21}=\mathrm{Z}_{12}$

$\mathrm{Z}_{22}=\frac{\mathrm{V}_{0}}{\mathrm{I}_{0}} \mid \mathrm{I}_{\mathrm{i} \rightarrow 0}=[\cos (\mu \mathrm{l})-\mathrm{BQ} \sin (\mu \mathrm{l})]$

$/\{\operatorname{Asin}(\mu \mathrm{l})-\mathrm{Q}[\cos (\mu \mathrm{l})+1]\}$

Where:

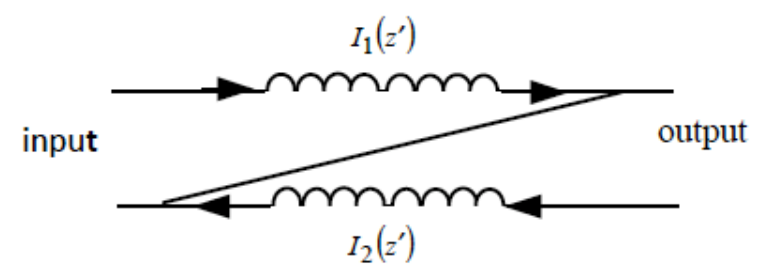

Fig. (4). 1:-1 transmission-line transformer.

$$
\begin{aligned}
& \mathrm{A}=\mathrm{j} /\left[\left(\mathrm{L}^{0.5} / \mathrm{C}^{0.5}\right)(1-\mathrm{K})^{0.5}\right], \\
& \mathrm{B}=\mathrm{j}\left(\mathrm{L}^{0.5} / \mathrm{C}^{0.5}\right)(1-\mathrm{K})^{0.5} / 2, \\
& \mathrm{Q}=-2 /\left[\mathrm{j} \beta\left(\mathrm{L}^{0.5} / \mathrm{C}^{0.5}\right)(1+\mathrm{K}) \ell\right] .
\end{aligned}
$$

By solving the above equation, the result can be obtained:

$$
Z_{\text {in }}=Z_{11}-\frac{Z_{12} Z_{21}}{R_{L}+Z_{22}}
$$

Where:

$\mathrm{R}_{\mathrm{L}}=$ the load impedance.

\section{A NETWORK MODEL FOR THE BIFILAR EVEN TRANSMISSION LINE}

$I_{1}\left(z^{\prime}\right)$ and $I_{2}\left(z^{\prime}\right)$, the currents in the two lines of bifilar transmission-line transformer, are not always equal. For example, in Fig. (4). (1:-1 transmission-line transformer), $I_{1}\left(z^{\prime}\right) \neq I_{2}\left(z^{\prime}\right)$. So, it cannot be analyzed by using transmission-line equation.

Fig. (5) shows the equivalent lumped-element circuit of a differential unit of transmission line. Its differential equation is obtained as follows:

$\frac{d U}{d z^{\prime}}=\frac{1}{2} Z\left(I_{1}+I_{2}\right)$

$\frac{d I_{1}}{d z^{\prime}}=\frac{d I_{2}}{d z^{\prime}}=Y U$

$\frac{d U_{\text {up }}}{d z^{\prime}}=\frac{1}{2} Z I_{1}$

Where: $Z=R+j \omega L, Y=G+j \omega C$.

From Eq. (21), we can get $I_{1}=I_{2}+2 C$ (assuming that $C$ is a complex constant).

Using the hypothesis $I=\frac{1}{2}\left(I_{1}+I_{2}\right)$, it is possible to define: $I_{1}=I+C$, and $I_{2}=I-C$.

So the solutions can be written as: 


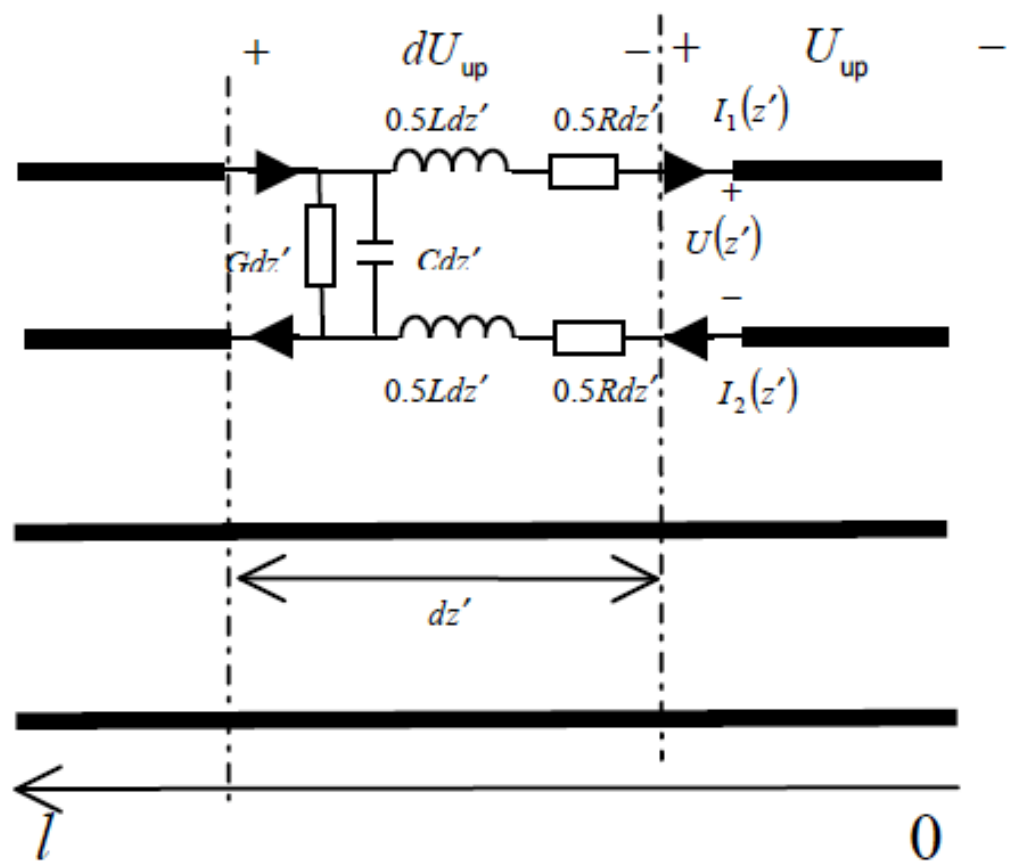

Fig. (5). The equivalent lumped-element circuit of a differential unit of transmission line.

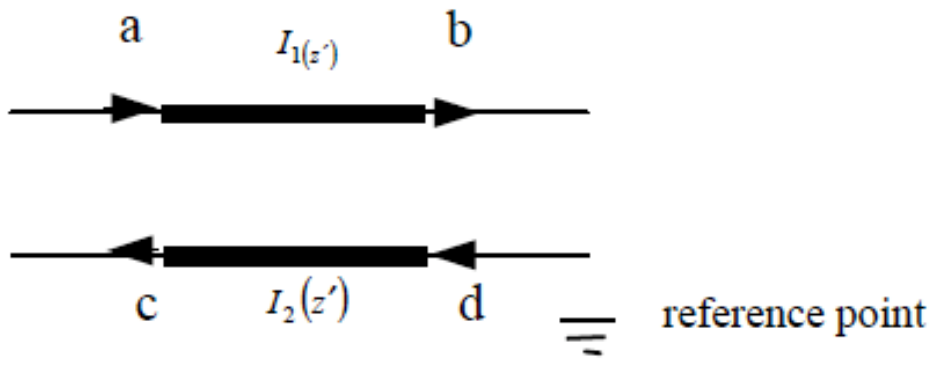

Fig. (6). A four-end network model for the asymmetrical (current) bifilar even transmission line.

$U=A e^{-r z^{\prime}}+B e^{r z^{\prime}}$

$I=\frac{1}{Z_{0}}\left(-A e^{-r z^{\prime}}+B e^{r z^{\prime}}\right)$

$U_{\text {up }}=\frac{1}{2} A\left(e^{-r z^{\prime}}-1\right)+\frac{1}{2} B\left(e^{r z^{\prime}}-1\right)+\frac{1}{2} C Z z^{\prime}$

Where:

$$
r=\sqrt{Z Y}=\alpha+j \beta \text {, and } Z_{0}=\sqrt{\frac{Z}{Y}} \text {. }
$$

$r=$ transmission coefficient,

$Z_{0}=$ characteristic impedance .

In Fig. (6), $I_{a}, I_{b}, I_{c}$ and $I_{d}$ are currents of the respective ends, while $U_{a}, U_{b}, U_{c}$ and $U_{d}$ are the voltages (to the reference point) of the respective ends. From Eq. (23), (24) and (25), we can obtain:

$$
\left[\begin{array}{c}
I_{a} \\
I_{b} \\
I_{c} \\
I_{d}
\end{array}\right]=\left[\begin{array}{c}
I_{1}(l) \\
I_{1}(0) \\
I_{2}(l) \\
I_{2}(0)
\end{array}\right]=\left[\begin{array}{ccc}
-\frac{1}{Z_{0}} e^{-r l} & \frac{1}{Z_{0}} e^{r l} & 1 \\
-\frac{1}{Z_{0}} & \frac{1}{Z_{0}} & 1 \\
-\frac{1}{Z_{0}} e^{-r l} & \frac{1}{Z_{0}} e^{r l} & -1 \\
-\frac{1}{Z_{0}} & \frac{1}{Z_{0}} & -1
\end{array}\right]\left[\begin{array}{l}
A \\
B \\
C
\end{array}\right]
$$

$$
\left[\begin{array}{l}
U_{b d} \\
U_{a c} \\
U_{a b}
\end{array}\right]=\left[\begin{array}{l}
U(0) \\
U(l) \\
U_{u p}(l)
\end{array}\right]=\left[\begin{array}{cccc}
0 & 1 & 0 & -1 \\
1 & 0 & -1 & 0 \\
1 & -1 & 0 & 0
\end{array}\right]\left[\begin{array}{c}
U_{a} \\
U_{b} \\
U_{c} \\
U_{d}
\end{array}\right]
$$

$$
=\left[\begin{array}{llc}
1 & 1 & 0 \\
e^{-r l} & e^{r l} & 0 \\
\frac{1}{2}\left(e^{-r l}-1\right) & \frac{1}{2}\left(e^{r l}-1\right) & \frac{1}{2} Z l
\end{array}\right]\left[\begin{array}{l}
A \\
B \\
C
\end{array}\right]
$$




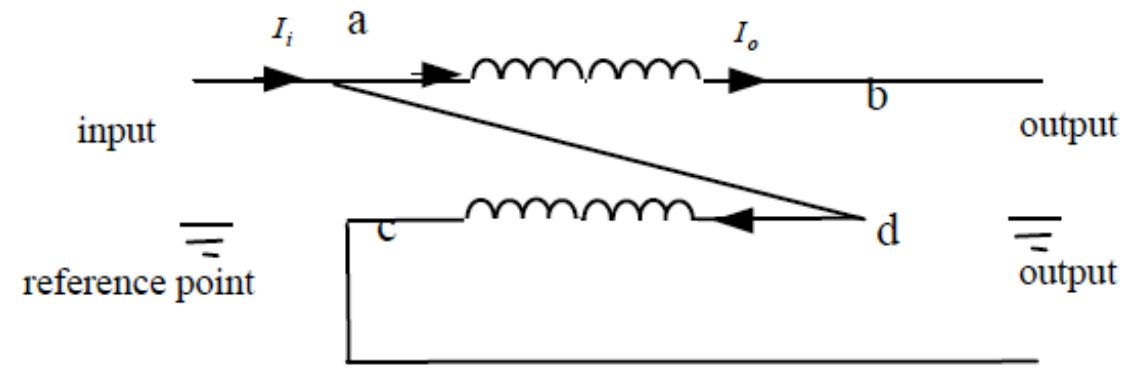

Fig. (7). The divider in multimode feed network.

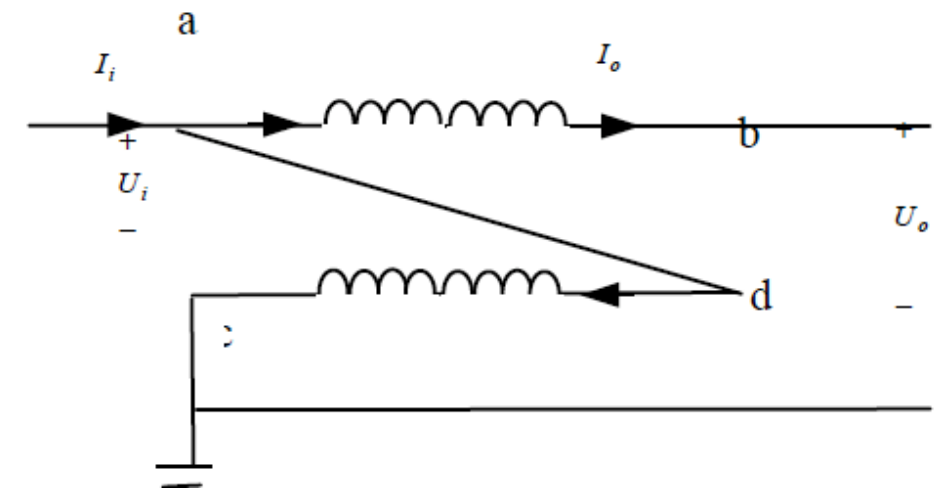

Fig. (8). The definite network model for divider shown in Fig.(7).

From Eq. (26) and (27), the current-voltage relationships of the four-end network model for the asymmetrical (current) bifilar even transmission line are derived as follows:

$$
\left[\begin{array}{l}
I_{a} \\
I_{b} \\
I_{c} \\
I_{d}
\end{array}\right]=\left\{\begin{array}{c}
\frac{1}{Z_{0}} \frac{1}{\sinh (r l)}\left[\begin{array}{cccc}
\cosh (r l) & -1 & -\cosh (r l) & 1 \\
1 & -\cosh (r l) & -1 & \cosh (r l) \\
\cosh (r l) & -1 & -\cosh (r l) & 1 \\
1 & -\cosh (r l) & -1 & \cosh (r l)
\end{array}\right] \\
+\frac{1}{Z l}\left[\begin{array}{cccc}
1 & -1 & 1 & -1 \\
1 & -1 & 1 & -1 \\
-1 & 1 & -1 & 1 \\
-1 & 1 & -1 & 1
\end{array}\right]
\end{array}\right]\left[\begin{array}{l}
U_{a} \\
U_{b} \\
U_{c} \\
U_{d}
\end{array}\right]
$$

When the currents in the two lines are equal $\left(I_{1}\left(z^{\prime}\right)=I_{2}\left(z^{\prime}\right)\right)$, Eq.(28) can be written as follows:

$$
\left[\begin{array}{c}
I_{a} \\
I_{b} \\
I_{c} \\
I_{d}
\end{array}\right]=\left\{\frac{1}{Z_{0}} \frac{1}{\sinh (r l)}\left[\begin{array}{cccc}
\cosh (r l) & -1 & -\cosh (r l) & 1 \\
1 & -\cosh (r l) & -1 & \cosh (r l) \\
\cosh (r l) & -1 & -\cosh (r l) & 1 \\
1 & -\cosh (r l) & -1 & \cosh (r l)
\end{array}\right]\right\}\left[\begin{array}{c}
U_{a} \\
U_{b} \\
U_{c} \\
U_{d}
\end{array}\right]
$$

By all appearances, Eq. (29) is one form of the solution of transmission-line equation.

\section{APPLICATION, ANALYSIS AND EXAMPLE}

Because of the different function, the two substructures have the different ends which can be connected together, the different input port and the different output port. In this paper, a divider is taken as an example. Connecting end a and $\mathrm{d}$ (Fig. 6), we can get divider which input from port a and output from port $b$ and $c$ (Fig. 7).

The function of divider in multimode feed network is to divide (or synthesize) the power and isolate the signal. Considering that divider is the three-port Indefinite network (no grounding), we can convert it into two-port definite network by connecting end $\mathrm{c}$ to ground, inputting from ac and outputting from bc (Fig. 8), So we can use two-port network to measure and analyze it conveniently.

Based on microwave network in conjunction with $U_{a}=U_{d}, U_{c}=0$, we can get:

$\left[\begin{array}{c}I_{i} \\ I_{o}\end{array}\right]=\left[\begin{array}{llll}1 & 0 & 0 & 1 \\ 0 & 1 & 0 & 0\end{array}\right]\left[\begin{array}{c}I_{a} \\ I_{b} \\ I_{c} \\ I_{d}\end{array}\right]$

$\left[\begin{array}{l}U_{i} \\ U_{o}\end{array}\right]=\left[\begin{array}{ll}1 & 0 \\ 0 & 1\end{array}\right]\left[\begin{array}{l}U_{a} \\ U_{b}\end{array}\right]$

By applying net cascade, Eq. (28), (30) and (31) can be combined as, (supposing transmission-line is loss free, so $r=j \beta)$ : 


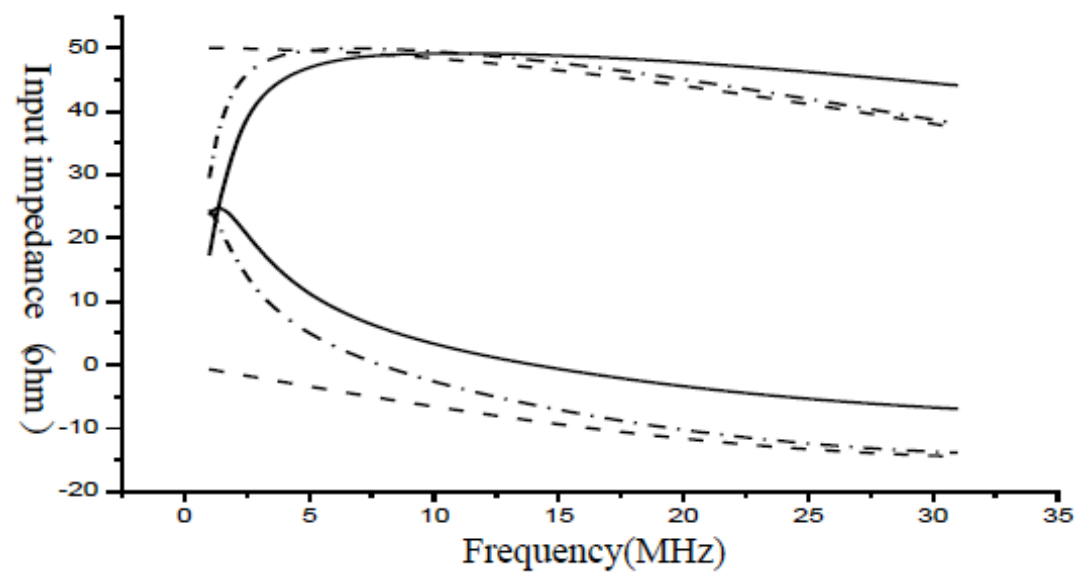

Fig. (9). Input impedance of the example.

$$
\left[\begin{array}{l}
I_{i} \\
-I_{o}
\end{array}\right]=\left\{\begin{array}{l}
\frac{1}{Z_{0}} \frac{1}{\sin (\beta l)}\left[\begin{array}{cc}
2(\cos (\beta l)+1) & -1-\cos (\beta l) \\
-1-\cos (\beta l) & \cos (\beta l)
\end{array}\right] \\
+\frac{1}{Z l}\left[\begin{array}{ll}
0 & 0 \\
0 & 1
\end{array}\right]
\end{array}\right\}\left\{\begin{array}{l}
U_{i} \\
U_{o}
\end{array}\right]
$$

Eq. (13) describes the Y-parameters of Definite network of divider (Fig. 8). Its input impedance can be written as:

$$
Z_{\text {in }}=\frac{\mathrm{R}_{\mathrm{L}}}{4} \times \frac{(1+\cos (\beta l))^{2}\left(1+\frac{\mathrm{R}_{\mathrm{L}}}{Z l}\right)+j\left[\begin{array}{l}
2 \frac{Z_{0}}{\mathrm{R}_{\mathrm{L}}}(1+\cos (\beta l)) \\
\left(1+\frac{\mathrm{R}_{\mathrm{L}}}{\mathrm{Zl}}\right)^{2}-\frac{\mathrm{R}_{\mathrm{L}}}{\mathrm{Z}_{0}} \cos (\beta l)
\end{array}\right] \sin (\beta l)}{(1+\cos (\beta l))^{2}\left(1+\frac{\mathrm{R}_{\mathrm{L}}}{\mathrm{Z} l}\right)^{2}+\frac{1}{4} \frac{\mathrm{R}_{\mathrm{L}}^{2}}{\mathrm{Z}_{0}^{2}} \sin ^{2}(\beta l)}
$$

Where: $R_{L}=$ the load connected with port $\mathrm{b}$ and $\mathrm{c}$. Referring to literature [2], when the frequency is not so high, the serial impedance of the two lines in the transmission-line transformer can be considered as $\mathrm{Zl}$. Taking coupling into account, we can obtain:

$$
Z l=4 Z_{\mathrm{p}}=4 \mathrm{j} \omega \mathrm{L}_{\mathrm{P}}=4 j \omega A_{L} N^{2}=4 j \omega \frac{\mu_{0} \mu_{e}}{C_{1}} \times 10^{3} \times N^{2}
$$

where: $z_{p}=$ parallel-reactance,

$L_{P}=$ magnetizing inductance,

$A_{L}=$ one-tune inductance,

$N=$ the number of tunes,

$\mu_{0}=$ the permeability of vacuum,

$\mu_{e}=$ the effective permeability of the media inside the coil,

$$
C_{1}=l_{e} / A_{e}=\text { dimension factor of magnetic core }\left(l_{e}=\right.\text { the }
$$
effective length of magnetic core, $A_{e}=$ the effective area of magnetic core).

\section{Example}

Divider to be exampled consists of eight tunes of coaxialline (characteristic impedance is $50 \mathrm{ohm}$ ) wound on a ferrite core, with outer and inner dimensions of $0.061 \mathrm{~m}$ and respectively. The core thickness is $0.015 \mathrm{~m}$.

Phase constant of coaxial-line is: $\beta=\frac{2 \pi f}{c} \sqrt{\varepsilon_{e f f}}$ [2] where : $\varepsilon_{\text {eff }}=$ the effective dielectric constant of the media inside the coil ( $\varepsilon_{e f f}$ of coaxial-line to be used in this example is 2.1[7]).

$j \omega A_{L}$ can be measured with HP4395A(all measures in this paper are done with HP4395A). And from Eq.(34), Zl can be computed (all computes in this paper are done with Matlab9). So, we can derive S-parameters and input impedance (with $R_{L}=200 \Omega$ ) by using Eq. (32) and Eq. (33) respectively. Fig. (9) provides results of measure and compute $(1 \sim 31 \mathrm{MHz})$.

Note: In Fig. (9), the real line, dashed and dash dot denote the metrical values, the values obtained with transmission-line equation and the values derived with the model in this paper respectively. In Fig. (9), the three lines above are the real part of input impedance, while the three lines below are the imaginary part.

\section{CONCLUSION}

Fig. (9) shows that at low frequency, the theoretical values obtained with the model in this paper fits into the metrical values better than the theoretical values derived from transmission-line equation, while at high frequency they are all consistent with the metrical values.

The substructure in multimode feed network is mainly wound with coaxial-line and twisted-pair. Coaxial-line has no magnetic flux leakage, coupling coefficients $=1$, preferable shield at both high frequency and low frequency. And, the calculation of its characteristic impedance and wavelength is ripe. So, it is feasible to analyze the substructure wound with coaxial-line by using the model of this paper. 


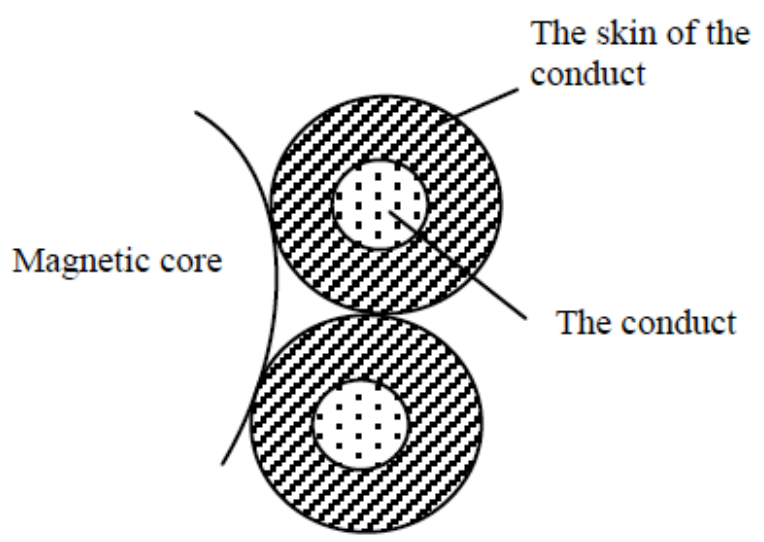

Fig.(10). Area of twisted-pair in the substructure.

For the substructure wound with twisted-pair, the problem is the calculation of the effective permeability $\left(\mu_{e}\right)$ and the effective dielectric constant ( $\varepsilon_{\text {eff }}$ ) of the media between the two conducts. As is shown in Fig. (10), the two conducts of twisted-pair do not cling each other and the interval between them is very small. So, the partly filled medium should be considered. Reference [2] provides a compute method. Only taking account of air and skin of the single conduct, it neglects the magnetic core.

\section{CONFLICT OF INTEREST}

The authors confirm that this article content has no conflict of interest.

\section{ACKNOWLEDGEMENTS}

This work is supported by National Natural Science Foundation of China (61077037), Key Scientific and Technological Project of Henan Province (102102210033), Science and Technology Research Project of The Education
Department of Henan Province (14B510019), National Training Programs of Innovation and Entrepreneurship for Undergraduates, Henan Normal University (201310476099).

\section{REFERENCES}

[1] S. Yang, "Short-wave miltimodemilti-feed antenna,"Research on Telecommunication Technology, vol. 8, pp. 14-19, Aug, 1994.

[2] J. Zhang, Broadband Ferrite Elements of Radiofrequency, Science Press, Beijing, 1986, pp. 80-92.

[3] C. Enzo, "Model characterizes transmission- line transformers," Microwaves \& RF, vol. 11, pp. 73-80, Nov, 1996

[4] H. Zhan, Y. Zhou, and Y. Zhang, "Property analysis of the usage in NiZn ferrite of broadband transmission-line transformer," High Power Laser and Particle Beams, vol. 22, no. 2, pp. 443-446, Feb, 2010.

[5] K.B. Niclas, R.R. Pereira, and A.P. Chang,"Transmission lines accurately model autotransformers,"Microwaves \& $R F$, vol. 11, pp. 67-67, Nov, 1992.

[6] K. Zhang, D. Li, Electromagnetic Theory for Microwaves and Optoelectronics, Publishing House of Electronics Industry, Beijing, 2001, pp. 118-126.

[7] H. Zhan, Z. Niu, and X. Du, "The theory research and design of feed network based on measurement database," In Proceedings of the ISTM Symposium (Conference) on Test and Measurement, Dalian, China, June 2005, pp. 7428-7431.

(C) Huawei et al.; Licensee Bentham Open.

This is an open access article licensed under the terms of the Creative Commons Attribution Non-Commercial License (http://creativecommons.org/licenses/by$\mathrm{nc} / 4.0 /$ ) which permits unrestricted, non-commercial use, distribution and reproduction in any medium, provided the work is properly cited. 